\title{
Mean Field Approximation for the Dense Charged Drop
}

\section{S. Bondarenko and K. Komoshvili}

Ariel University, Ariel, Israel

Correspondence should be addressed to S. Bondarenko; sergeyb@ariel.ac.il

Received 22 October 2017; Revised 29 November 2017; Accepted 6 December 2017; Published 31 December 2017

Academic Editor: Edward Sarkisyan-Grinbaum

Copyright (C) 2017 S. Bondarenko and K. Komoshvili. This is an open access article distributed under the Creative Commons Attribution License, which permits unrestricted use, distribution, and reproduction in any medium, provided the original work is properly cited. The publication of this article was funded by SCOAP ${ }^{3}$.

We consider in this note the mean field approximation for the description of the probe charged particle in a dense charged drop. We solve the corresponding Schrödinger equation for the drop with spherical symmetry in the first order of mean field approximation and discuss the obtained results.

\section{Introduction}

Collisions of relativistic nuclei in the RHIC and LHC experiments at very high energies led to the discovery of a new state of matter named quark-gluon plasma (QGP). At the initial stages of the scattering, this plasma resembles almost an ideal liquid whose microscopic structure is not yet well understood [1-11]. The data obtained in the RHIC experiments is in good agreement with the predictions of the ideal relativistic fluid dynamics [12-32], which establishes fluid dynamics as the main theoretical tool to describe collective flow in collisions. As an input to the hydrodynamical evolution of the particles, it is assumed that, after a very short time, $\tau<1 \mathrm{fm} / \mathrm{s}[31,32]$, the matter reaches a thermal equilibrium and expands with a very small shear viscosity [33-35].

In this paper, we continue to develop the model proposed in [36-39]. Namely, we assume that, at the local energy density fluctuations, hot drops [40-42] are created at the very initial stage of interactions at times $\tau<0.5 \mathrm{fm}$. These fireballs (hot drops) are very dense and small, their size is much smaller than the proton size (see [36-39]), and their energy density is much larger than the density achieved in high-energy interactions at the energies $\sqrt{s}<100 \mathrm{GeV}$. In our model, we assume that the fireballs consist of particles with weak interparticle interactions and have a nonzero charge. We consider this drop of charged particles from the point of view of quantum statistical physics. The most general Hamiltonian for this system can be written in the form which describes all possible interactions between the particles in the drop:

$$
H=H^{0}+V^{1}+V^{2}+\cdots+V^{i}+\cdots
$$

(see [43]), where as usual $V^{1}$ is the energy of interaction of the particle with an external field, $V^{2}$ is the energy of pairlike interactions, and so forth. The mean field approximation for the probe particle in the system of charged particles, therefore, can be introduced by the following perturbative scheme. First of all, we can consider the motion of only one probe particle in the mean field of all other particles, which corresponds to preserving only the $V^{1}$ term in the expression of (1). This approximation will lead to the modification of the propagator of the particle, namely, from a free propagator to some "dressed" one. At the next step, we can take two probe particles, each of which will propagate in the mean field of the other charges of the system, similar to the first approximation, but additionally we can introduce the interaction of these two particles one with another in the mean field of the remaining charges in the drop, which requires introduction of one $V^{2}$ term in the expression of (1) in the mean field approximation. Further, we can increase the number of the probe particles in the system, considering, in addition to pair interactions, the interactions of free probe particles and so on.

In the present calculations, we limit ourselves to the first order of the mean field approach; namely, we will consider the motion of one nonrelativistic probe particle in the external mean field created by all other particles in the charged drop. 


\section{Mean Field Approximation for the Hamiltonian of the System}

In the absence of the external field, we write the Hamiltonian of the system of charged particles as

$$
\begin{aligned}
H & =-\frac{1}{2 m} \int \Psi_{\alpha}^{+}(t, \vec{r}) \Delta \Psi_{\alpha}(t, \vec{r}) d^{3} x-\mu N+\frac{1}{2} \\
& \cdot \int \Psi_{\beta}^{+}(t, \vec{r}) \Psi_{\alpha}^{+}\left(t, \vec{r}^{\prime}\right) U\left(\vec{r}-\vec{r}^{\prime}\right) \Psi_{\alpha}\left(t, \vec{r}^{\prime}\right) \\
& \cdot \Psi_{\beta}(t, \vec{r}) d^{3} x d^{3} x^{\prime} .
\end{aligned}
$$

Here,

$$
N=\int \Psi_{\alpha}^{+}(t, \vec{r}) \Psi_{\alpha}(t, \vec{r}) d^{3} x
$$

is a particle number operator. Considering the mean field approximation for a spherically symmetrical system, we introduce

$$
\begin{aligned}
\Psi_{\alpha}^{+}\left(t, \vec{r}^{\prime}\right) \Psi_{\beta}(t, \vec{r}) & \approx\left\langle\Psi_{\alpha}^{+}\left(t, \vec{r}^{\prime}\right) \Psi_{\beta}(t, \vec{r})\right\rangle \\
& =\delta_{\alpha \beta} f\left(r, r^{\prime}, r_{0}\right)
\end{aligned}
$$

as some particles density for the droplet with characteristic size $r_{0}$; here, $r=|\vec{r}|$. The $f\left(r, r^{\prime}, r_{0}\right)$ function is a distribution function of the system of interest; it can be correctly determined by writing the corresponding Vlasov or Boltzmann equations coupled to system (1). In our case, we will not consider a particular form of this function, but instead we will discuss its form based on some physical assumptions only. Therefore, we obtain the following for the Hamiltonian:

$$
\begin{aligned}
H & =-\frac{1}{2 m} \int \Psi_{\alpha}^{+}(t, \vec{r}) \Delta \Psi_{\alpha}(t, \vec{r}) d^{3} x-\mu N+\frac{1}{2} \\
& \cdot \int \Psi_{\alpha}^{+}(t, \vec{r}) U\left(\vec{r}-\vec{r}^{\prime}\right) f\left(r, r^{\prime}, r_{0}\right) \\
& \cdot \Psi_{\alpha}\left(t, \vec{r}^{\prime}\right) d^{3} x d^{3} x^{\prime},
\end{aligned}
$$

which represents now the energy of the probe particle in the mean field created by the other particles of the system. Due to the spherical symmetry of the problem, we expand all the operators in the Hamiltonian expression in terms of spherical harmonic functions. We have the following for the two-particle interaction potential:

$$
\begin{aligned}
& U\left(\vec{r}-\vec{r}^{\prime}\right)=\sum_{l=0}^{\infty} \sum_{m=-l}^{l} \frac{4 \pi q^{2}}{2 l+1}\left(\theta\left(r^{\prime}-r\right) \frac{r^{l}}{\left(r^{\prime}\right)^{l+1}}\right. \\
& \left.+\theta\left(r-r^{\prime}\right) \frac{r^{\prime l}}{r^{l+1}}\right) Y_{l m}^{*}(\Psi, \Phi) Y_{l m}(\psi, \phi)
\end{aligned}
$$

with $\Psi, \Phi$ as spherical angles of $r^{\prime}$ vector, $\psi, \phi$ as spherical angles of $r$ vector in some spherical coordinate system, and $\theta(r)$ as the step function. Correspondingly, we write the particle-field operator as

$$
\Psi_{\alpha}(t, \vec{r})=\sum_{l=0}^{\infty} \sum_{m=-l}^{l} \psi_{\alpha l m}(t, r) Y_{l m}(\psi, \phi) .
$$

Using the orthogonality property of the harmonic functions

$$
\int Y_{l m}(\psi, \phi) Y_{l^{\prime} m^{\prime}}^{*}(\psi, \phi) d \Omega=\delta_{l l^{\prime}} \delta_{m m^{\prime}}
$$

with $d^{3} x=r^{2} d r d \Omega$, we rewrite the Hamiltonian equation (5) in a one-dimensional form as a function of $r$ and $r^{\prime}$ only:

$$
\begin{aligned}
H & =-\frac{1}{2 m} \sum_{l=0}^{\infty} \sum_{m=-l}^{l} \int_{0}^{\infty}\left(\psi_{\alpha l m}^{+}(t, r) \Delta_{r} \psi_{\alpha l m}(t, r)-\frac{l(l+1)}{r^{2}} \psi_{\alpha l m}^{+}(t, r) \psi_{\alpha l m}(t, r)\right) r^{2} d r \\
& -\mu \sum_{l=0}^{\infty} \sum_{m=-l}^{l} \int_{0}^{\infty} \psi_{\alpha l m}^{+}(t, r) \psi_{\alpha l m}(t, r) r^{2} d r+\sum_{l=0}^{\infty} \sum_{m=-l}^{l} \frac{4 \pi q^{2}}{2 l+1} \\
& \cdot \int_{0}^{\infty} r^{2} d r\left(\int_{r}^{\infty} \frac{r^{l}}{r^{\prime(l+1)}} f\left(r, r^{\prime}, r_{0}\right) \psi_{\alpha l m}^{+}(t, r) \psi_{\alpha l m}\left(t, r^{\prime}\right) r^{\prime 2} d r^{\prime}+\int_{0}^{r} \frac{r^{\prime l}}{r^{(l+1)}} f\left(r, r^{\prime}, r_{0}\right) \psi_{\alpha l m}^{+}(t, r) \psi_{\alpha l m}\left(t, r^{\prime}\right) r^{\prime 2} d r^{\prime}\right) .
\end{aligned}
$$

In the next section, we solve Schrödinger's equation corresponding to this Hamiltonian.

\section{Equations of Motion}

We introduce the usual commutation relations for the fields of interest (see (7)):

$$
\left\{\Psi_{\alpha}(t, \vec{r}), \Psi_{\beta}^{+}\left(t, \vec{r}^{\prime}\right)\right\}=\delta_{\alpha \beta} \delta^{3}\left(\vec{t}-\vec{r}^{\prime}\right) .
$$

Using the property of (8), we correspondingly obtain onedimensional commutation relations for the new fields:

$$
\left\{\psi_{\alpha l m}(t, r), \psi_{\beta l^{\prime} m^{\prime}}^{+}\left(t, r^{\prime}\right)\right\}=\frac{1}{r^{2}} \delta_{\alpha \beta} \delta_{l l^{\prime}} \delta_{m m^{\prime}} \delta\left(r-r^{\prime}\right) .
$$


The Schrödinger equation for $\psi_{\alpha l m}(t, r)$ field, therefore, has the following form:

$$
\begin{aligned}
& \iota \frac{\partial}{\partial t} \psi_{\alpha l m}(t, r)=\left(-\frac{1}{2 m}\left(\Delta_{r}-\frac{l(l+1)}{r^{2}}\right)-\mu\right) \\
& \cdot \psi_{\alpha l m}(t, r) \\
& +\frac{4 \pi q^{2}}{2 l+1}\left(\int_{r}^{\infty} \frac{r^{l}}{r^{\prime(l+1)}} f\left(r, r^{\prime}, r_{0}\right) \psi_{\alpha l m}\left(t, r^{\prime}\right) r^{\prime 2} d r^{\prime}\right. \\
& \left.+\int_{0}^{r} \frac{r^{\prime l}}{r^{(l+1)}} f\left(r, r^{\prime}, r_{0}\right) \psi_{\alpha l m}\left(t, r^{\prime}\right) r^{\prime 2} d r^{\prime}\right) .
\end{aligned}
$$

Rescaling the drop's density function and rewriting it in the dimensionless form as

$$
f\left(r, r^{\prime}, r_{0}\right) \longrightarrow \frac{1}{r^{3 / 2}\left(r^{\prime}\right)^{3 / 2}} f\left(r, r^{\prime}, r_{0}\right),
$$

introducing a new variable in integrals in (12),

$$
r^{\prime}=x r
$$

we rewrite the integrals in (12) finally as

$$
\begin{aligned}
& \frac{4 \pi}{r(2 l+1)}\left(\int_{1}^{\infty} \frac{d x}{x^{l+1 / 2}} f\left(r, x, r_{0}\right) \psi_{\alpha l m}(t, x)\right. \\
& \left.\quad+\int_{0}^{1} d x x^{l+1 / 2} f\left(r, x, r_{0}\right) \psi_{\alpha l m}(t, x)\right) .
\end{aligned}
$$

For the case of the drop of small size, we can expand our $\psi$ function in (15) around $x=1$ in both terms; this point gives the main contribution to both integrals. Therefore, in the first approximation, we have the following for (15):

$$
\begin{aligned}
& \frac{4 \pi}{r(2 l+1)}\left(\int_{1}^{\infty} \frac{d x}{x^{l+1 / 2}} f\left(r, x, r_{0}\right) \psi_{\alpha l m}(t, x r)\right. \\
& \left.+\int_{0}^{1} d x x^{l+1 / 2} f\left(r, x, r_{0}\right) \psi_{\alpha l m}(t, x r)\right) \\
& \approx \psi_{\alpha l m}(t, r) \frac{4 \pi}{r(2 l+1)}\left(\int_{1}^{\infty} \frac{d x}{x^{l+1 / 2}} f\left(r, x, r_{0}\right)\right. \\
& \left.+\int_{0}^{1} d x x^{l+1 / 2} f\left(r, x, r_{0}\right)\right)=\frac{Q_{l}(r)}{r} \psi_{\alpha l m}(t, r)
\end{aligned}
$$

with $Q_{l}$ as an $l$ multipole moment of the drop. The Schrödinger equation (12) now acquires the following form:

$$
\begin{aligned}
& \iota \frac{\partial}{\partial t} \psi_{\alpha l m}(t, r) \\
& \quad=\left(-\frac{1}{2 m}\left(\Delta_{r}-\frac{l(l+1)}{r^{2}}\right)-\mu+\frac{q^{2}}{r} Q_{l}(r)\right) \\
& \quad \cdot \psi_{\alpha l m}(t, r) .
\end{aligned}
$$

Representing the wave function as

$$
\psi_{\alpha l m}(t, r)=u_{\alpha l m}(r) e^{-\iota t(E-\mu)}
$$

we obtain the Schrödinger equation for the particle in the following form:

$$
\left(\frac{1}{2 m} \Delta_{r}-\frac{1}{2 m} \frac{l(l+1)}{r^{2}}+E-\frac{q^{2}}{r} Q_{l}(r)\right) u_{\alpha l m}(r)=0 .
$$

In general, we cannot solve this equation without knowledge of the form of $f\left(r, x, r_{0}\right)$ particles distribution function in integrals of (15). Nevertheless, we can guess the form of the function in the $r \leq r_{0}$ region of the drop, mostly interesting for us. Indeed, at $r \gg r_{0}$, which is outside the drop region, the potential equation (16) is the usual Coulomb potential, but in the $r \leq r_{0}$ region, the situation is different. The existence of the drop requires the presence of some potential well at $r \leq r_{0}$ which will keep particles inside the drop for some (very short) time and, therefore, it must be the potential's minimum present somewhere between $r=0$ and $r \propto r_{0}$. Hence, this minimum is the indication of the creation of the dense drop of finite size in the interaction system of interest and, consequently, we can write the potential energy from (19) in this region as

$$
\begin{aligned}
& \frac{1}{2 m} \frac{l(l+1)}{r^{2}}+\frac{Q_{l}(r) q^{2}}{r} \\
& \approx \frac{1}{2 m} \frac{l(l+1)}{r_{\text {min }}^{2}}+\frac{Q_{l}\left(r_{\min }\right) q^{2}}{r_{\text {min }}} \\
& \quad+\frac{A_{l}\left(r_{\text {min }}\right) q^{2}}{2 r_{0}^{3}}\left(r-r_{\text {min }}\right)^{2},
\end{aligned}
$$

where we assumed that the potential energy acquires its minimum at $r_{\text {min }} ; A_{l}\left(r_{\text {min }}\right)$ here are the positive coefficients of the potential's expansion around this minimum. This situation, in fact, is similar to the situation in the system of two-atom molecules (see [44] and the references therein), where two atoms are kept inside some mutual potential well. Inserting the expansion of (20) in (19), we obtain the following equation:

$$
\begin{gathered}
\left(\frac{1}{2 m} \Delta_{r}+E-\frac{1}{2 m} \frac{l(l+1)}{r_{\min }^{2}}-Q_{l}\left(r_{\min }\right) \frac{q^{2}}{r_{\min }}\right. \\
\left.-A_{l}\left(r_{\min }\right) \frac{q^{2}}{2 r_{0}^{3}}\left(r-r_{\min }\right)^{2}\right) u_{\alpha l m}(r)=0 .
\end{gathered}
$$

The solution of this equation is similar to the solution of the Schrödinger equation for the harmonic oscillator with energy levels defined by

$$
E^{\prime}=E-\frac{1}{2 m} \frac{l(l+1)}{r_{\min }^{2}}-Q_{l}\left(r_{\min }\right) \frac{q^{2}}{r_{\min }}
$$

and consequently the energy levels of the system are given by

$$
\begin{aligned}
E_{n l}= & \frac{1}{2 m} \frac{l(l+1)}{r_{\min }^{2}}+Q_{l}\left(r_{\min }\right) \frac{q^{2}}{r_{\min }} \\
& +q\left(\frac{A_{l}\left(r_{\min }\right)}{m r_{0}^{3}}\right)^{1 / 2}\left(n+\frac{1}{2}\right) \quad n=0,1,2, \ldots,
\end{aligned}
$$


with the wave functions

$$
u_{\alpha l m}(r)=c_{\alpha l m} \frac{e^{-\left(r-r_{\min }\right)^{2} /\left(2 R^{2}\right)}}{r \sqrt{R}} H_{n}\left(\frac{\left(r-r_{\min }\right)}{R}\right),
$$

where

$$
R=\left(\frac{r_{0}^{3}}{m A_{l}\left(r_{\min }\right) q^{2}}\right)^{1 / 4} .
$$

We note that the obtained solution is indeed similar to the solution of the Schrödinger equation for the two-atom molecules (see, e.g., [44]).

\section{Conclusion}

In this note, we demonstrated that the spectrum of the charged nonrelativistic particle in the dense charged drop has a quantum structure (see (23)), and it is determined by three terms in the first mean field approximation. The first term in (23) can be considered as the quantized rotation energy of the drop; the second one is the quantized electrostatic energy due to the multipole moments of the charged drop. The third term in the expression of (23) is the usual quantum corrections to the energy due to the oscillation of the particle inside the drop with eigenfrequencies determined by the form of the distribution function of the particles in the drop. The presence of this minimum is a necessary condition of the drop's creation (see also [36-39]). The values of these corrections have an additional degeneracy of energy levels defined by $l$ quantum number in comparison to the ordinary quantum oscillator. In this formulation, the considered problem is similar to the problem of the description of the system of the two-atom molecule (see [44]) (we note that the proposed approach can be used also for the description of bound states created at low energy interactions, and we plan to investigate this subject in a separate publication). Further development of the approach can include the consideration of higher orders of mean field approximation for the system and introduction of the kinetic equation for the distribution function of (4) coupled to the Hamiltonian; we plan to consider these problems in the following publications.

We conclude that our model can be useful for the clarification of the spectrum of the produced particles, which is influenced by the quantum-mechanical properties of the QCD fireball. We believe that this approach will provide the connection between the data, obtained in high-energy collisions of protons and nuclei in the LHC and RHIC experiments [12-32, 45-49], and microscopic fields inside the collision region.

\section{Conflicts of Interest}

The authors declare that there are no conflicts of interest regarding the publication of this paper.

\section{References}

[1] E. Shuryak, "Four lectures on strongly coupled Quark Gluon Plasma," Nuclear Physics B, vol. 195, pp. 111-156, 2009.
[2] E. Shuryak, "Physics of strongly coupled quark-gluon plasma," Progress in Particle and Nuclear Physics, vol. 62, no. 1, pp. 48-101, 2009.

[3] E. V. Shuryak and I. Zahed, "Toward a theory of binary bound states in the quark-gluon plasma," Physical Review D: Particles, Fields, Gravitation and Cosmology, vol. 70, Article ID 054507, 2004.

[4] B. Berdnikov and K. Rajagopal, "Slowing out of equilibrium near the QCD critical point," Physical Review D: Particles, Fields, Gravitation and Cosmology, vol. 61, Article ID 105017, 2000.

[5] V. Koch, A. Majumder, and J. Randrup, "Baryon-strangeness correlations: a diagnostic of strongly interacting matter," Physical Review Letters, vol. 95, Article ID 182301, 2005.

[6] J. Liao and E. V. Shuryak, "What do lattice baryonic susceptibilities tell us about quarks, diquarks, and baryons at $T>T_{c}$," Physical Review D: Particles, Fields, Gravitation and Cosmology, vol. 73, Article ID 014509, 2006.

[7] M. Nahrgang, C. Herold, and M. Bleicher, "Influence of an inhomogeneous and expanding medium on signals of the QCD phase transition," Nuclear Physics A, vol. 904-905, pp. 899c902c, 2013.

[8] J. Steinheimer and J. Randrup, "Spinodal Amplification of Density Fluctuations in Fluid-Dynamical Simulations of Relativistic Nuclear Collisions," Physical Review Letters, vol. 109, Article ID 212301, 2012.

[9] V. V. Skokov and D. N. Voskresensky, "Hydrodynamical description of first-order phase transitions: Analytical treatment and numerical modeling," Nuclear Physics A, vol. 828, no. 3-4, pp. 401-438, 2009.

[10] V. V. Skokov and D. N. Voskresensky, "Hydrodynamical description of a hadron-quark first-order phase transition," JETP Letters, vol. 90, no. 4, pp. 223-227, 2009.

[11] J. Randrup, "Phase transition dynamics for baryon-dense matter," Physical Review C: Nuclear Physics, vol. 79, Article ID 054911, 2009.

[12] T. Ludlam and S. Aronson, "BRAHMS and STAR and PHOBOS and PHENIX collaborations," Tech. Rep. BNL-73847-2005, 2005.

[13] K. Adcox, S. S. Adler, S. Afanasiev et al., "Formation of dense partonic matter in relativistic nucleus-nucleus collisions at RHIC: experimental evaluation by the PHENIX collaboration," Nuclear Physics A, vol. 757, no. 1-2, pp. 184-283, 2005.

[14] J. Adams, M. M. Aggarwala, Z. Ahammed et al., "Experimental and theoretical challenges in the search for the quark-gluon plasma: the STAR Collaboration's critical assessment of the evidence from RHIC collisions," Nuclear Physics A, vol. 757, no. 1-2, pp. 102-183, 2005.

[15] I. Arsene, I. G. Bearden, D. Beavis et al., "Quark-gluon plasma and color glass condensate at RHIC? The perspective from the BRAHMS experiment," Nuclear Physics A, vol. 757, no. 1-2, pp. $1-27,2005$.

[16] C. Jena, in Proceedings of the DAE Symposium on Nuclear Physics, vol. 56, pp. 1180-1181, 2011.

[17] A. Jipa, O. Ristea, C. Ristea et al., "New information on the dynamics of relativistic nucleus-nucleus collisions," Journal of Physics: Conference Series, vol. 381, Article ID 012042, 2012.

[18] P. Steinberg, "What have we learned about the quark-gluon plasma with the ATLAS detector at the LHC?" Nuclear Physics A, vol. 932, pp. 9-16, 2014.

[19] J. Velkovska, "What have hard probes taught us about the quark-gluon plasma as measured in CMS?" Nuclear Physics A, vol. 932, pp. 17-24, 2014. 
[20] I. Arsene, "BRAHMS collaboration results for relativistic heavy ion collisions," Physics of Particles and Nuclei, vol. 39, no. 7, pp. 1082-1086, 2008.

[21] M. Przybycien, "Recent results on soft probes of the QuarkGluon Plasma from the ATLAS experiment at the LHC," Nuclear and Particle Physics Proceedings, vol. 273-275, pp. 1539$1545,2016$.

[22] J. Adam, D. Adamová, M. M. Aggarwal et al., "Production of light nuclei and anti-nuclei in $p p$ and $\mathrm{Pb}-\mathrm{Pb}$ collisions at energies available at the CERN Large Hadron Collider," Physical Review C, vol. 93, Article ID 024917, 2016.

[23] M. Chojnacki, "Soft probes of the Quark-Gluon Plasma measured by ALICE," Nuclear and Particle Physics Proceedings, vol. 273-275, pp. 1553-1558, 2016.

[24] CMS Collaboration, "Pseudorapidity distributions of charged hadrons in proton-lead collisions at $\sqrt{s_{N N}}=5.02$ and $8.16 \mathrm{TeV}$," Tech. Rep. CMS-PAS-HIN-16-021, 2017.

[25] B. Wosiek, "Measurements of hard probes of the quark-gluon plasma with the ATLAS experiment at the LHC," Nuclear and Particle Physics Proceedings, vol. 273-275, pp. 1546-1552, 2016.

[26] A. M. Sirunyan, W. Adam, F. Ambrogi, E. Asilar et al., "Pseudorapidity distributions of charged hadrons in proton-lead collisions at $\sqrt{s_{N N}}=5.02$ and $8.16 \mathrm{TeV}, 2$ 2017, https://arxiv.org/abs/ 1710.09355 .

[27] A. Barbano, "Heavy flavours as a probe for the Quark-Gluon Plasma with ALICE at the LHC," IL NUOVO CIMENTO C, vol. 40, no. 1, p. 27, 2017.

[28] B. Huang, "Overview of Recent Results from the STAR experiment," Nuclear and Particle Physics Proceedings, vol. 289-290, pp. 19-24, 2017.

[29] P. F. Kolb, P. Huovinen, U. Heinz, and H. Heiselberg, "Elliptic flow at SPS and RHIC: from kinetic transport to hydrodynamics," Physics Letters B, vol. 500, no. 3-4, pp. 232-240, 2001.

[30] D. Teaney, J. Lauret, and E. V. Shuryak, "Flow at the SPS and RHIC as a quark-gluon plasma signature," Physical Review Letters, vol. 86, no. 21, pp. 4783-4786, 2001.

[31] U. W. Heinz and P. F. Kolb, "Early thermalization at RHIC," Nuclear Physics A, vol. 702, no. 1-4, pp. 269-280, 2002.

[32] A. Peshier and W. Cassing, "The hot nonperturbative gluon plasma is an almost ideal colored liquid," Physical Review Letters, vol. 94, Article ID 172301, 2005.

[33] P. Romatschke and U. Romatschke, "Viscosity information from relativistic nuclear collisions: how perfect is the fluid observed at RHIC?" Physical Review Letters, vol. 99, no. 17, Article ID 172301, 2007.

[34] B. Schenke, S. Jeon, and C. Gale, "Higher flow harmonics from," Physical Review C: Nuclear Physics, vol. 85, Article ID 024901, 2012.

[35] D. Teaney, "Effect of shear viscosity on spectra, elliptic flow, and Hanbury Brown-Twiss radii," Physical Review C: Nuclear Physics, vol. 68, Article ID 034913, 2003.

[36] S. Bondarenko and K. Komoshvili, "Gas-liquid transition in the model of particles interacting at high energy," The European Physical Journal C, vol. 73, article 2624, 2013.

[37] S. Bondarenko and K. Komoshvili, "Transverse transport properties of a charged drop in an electric field," International Journal of Modern Physics E, vol. 24, no. 5, Article ID 1550034, 23 pages, 2015.

[38] S. Bondarenko, K. Komoshvili, and A. Prygarin, "Transport properties of a charged fireball in an external electromagnetic field," Nuclear Physics A, vol. 950, pp. 129-162, 2016.
[39] S. Bondarenko and K. Komoshvili, "Transverse kinetics of a charged drop in an external electric," AIP Conference Proceedings, vol. 1701, Article ID 060007, 2016.

[40] M. Gyulassy, D. H. Rischke, and B. Zhang, "Hot spots and turbulent initial conditions of quark-gluon plasmas in nuclear collisions," Nuclear Physics A, vol. 613, no. 4, pp. 397-434, 1997.

[41] G. Torrieri, "Quark fluids in heavy ion collisions," 2009, https://arxiv.org/abs/0911.5479.

[42] M. Stephanov, K. Rajagopal, and E. Shuryak, "Event-by-event fluctuations in heavy ion collisions and the QCD critical point," Physical Review D: Particles, Fields, Gravitation and Cosmology, vol. 60, Article ID 114028, 1999.

[43] L. D. Landau, E. M. Lifshitz, and L. P. Pitaevskii, Statistical Physics, Part 1 (Course Theoretical Physics, Volume 5), Pergamon Press, 1980.

[44] M. H. Jeremy, "An Introduction to the dynamics of Van der Waals molecules," Advances in Molecular Vibrations and Collision Dynamics 1A, vol. 1, 1990.

[45] D. Kharzeev, E. Levin, and K. Tuchin, "Multiparticle production and thermalization in high-energy QCD," Physical Review C: Nuclear Physics, vol. 75, Article ID 044903, 2007.

[46] E. K. G. Sarkisyan and A. S. Sakharov, "Relating multihadron production in hadronic and nuclear collisions," The European Physical Journal C, vol. 70, no. 3, pp. 533-541, 2010.

[47] A. N. Mishra, R. Sahoo, E. K. G. Sarkisyan, and A. S. Sakharov, "Effective-energy budget in multiparticle production in nuclear collisions," The European Physical Journal C, vol. 74, article 3147, 2014, Erratum: [The European Physical Journal C 75, 70 (2015)].

[48] E. K. G. Sarkisyan, A. N. Mishra, R. Sahoo, and A. S. Sakharov, "Multihadron production dynamics exploring the energy balance in hadronic and nuclear collisions," Physical Review D: Particles, Fields, Gravitation and Cosmology, vol. 93, Article ID 079904, 2016, Addendum: [Physical Review D: Particles, Fields, Gravitation and Cosmology 93, no. 7, 079904 (2016)].

[49] E. K. G. Sarkisyan, A. N. Mishra, R. Sahoo, and A. S. Sakharov, "Centrality dependence of midrapidity density from $\mathrm{GeV}$ to $\mathrm{TeV}$ heavy-ion collisions in the effective-energy universality picture of hadroproduction," Physical Review D: Particles, Fields, Gravitation and Cosmology, vol. 94, Article ID 011501(R), 2016. 

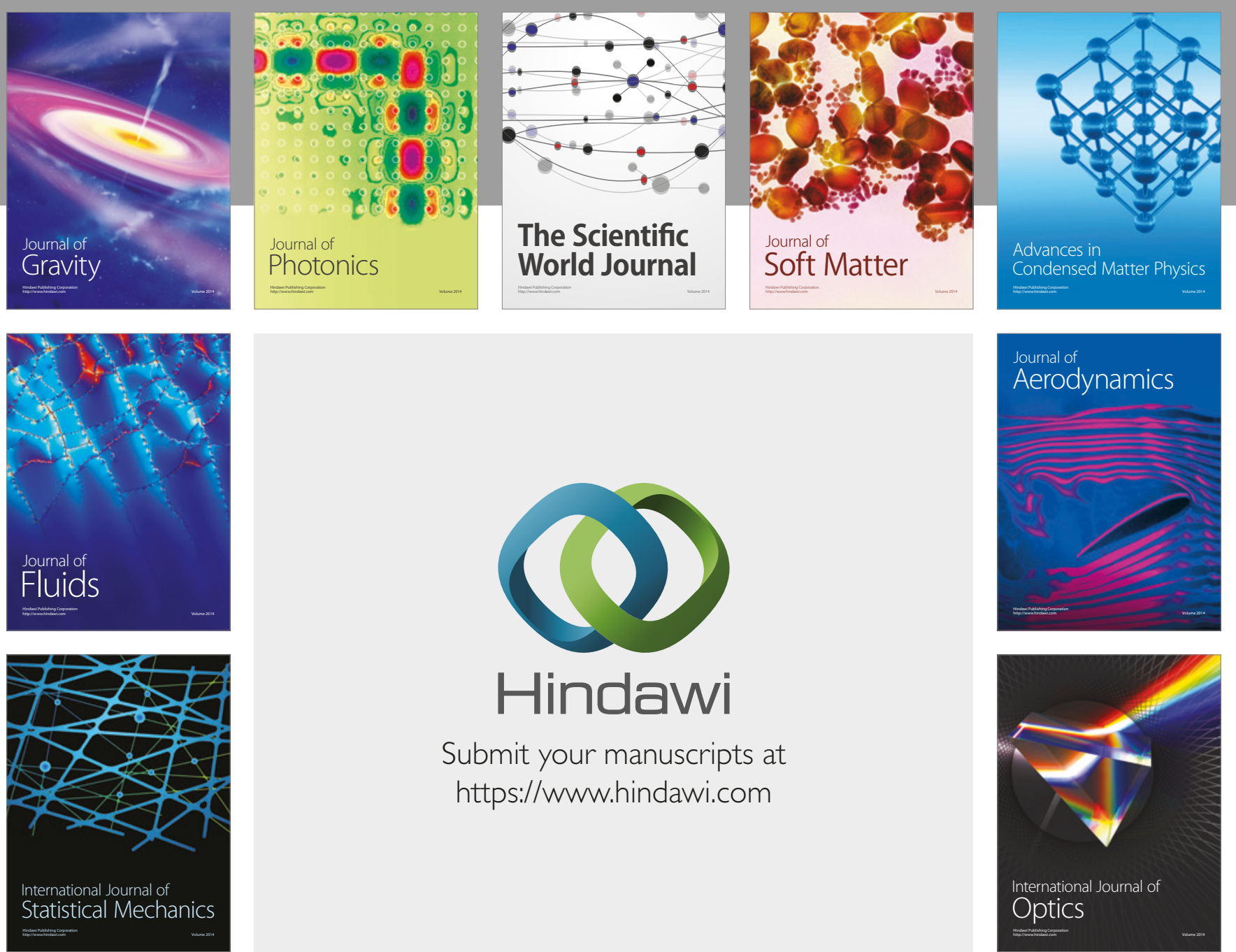

Submit your manuscripts at

https://www.hindawi.com
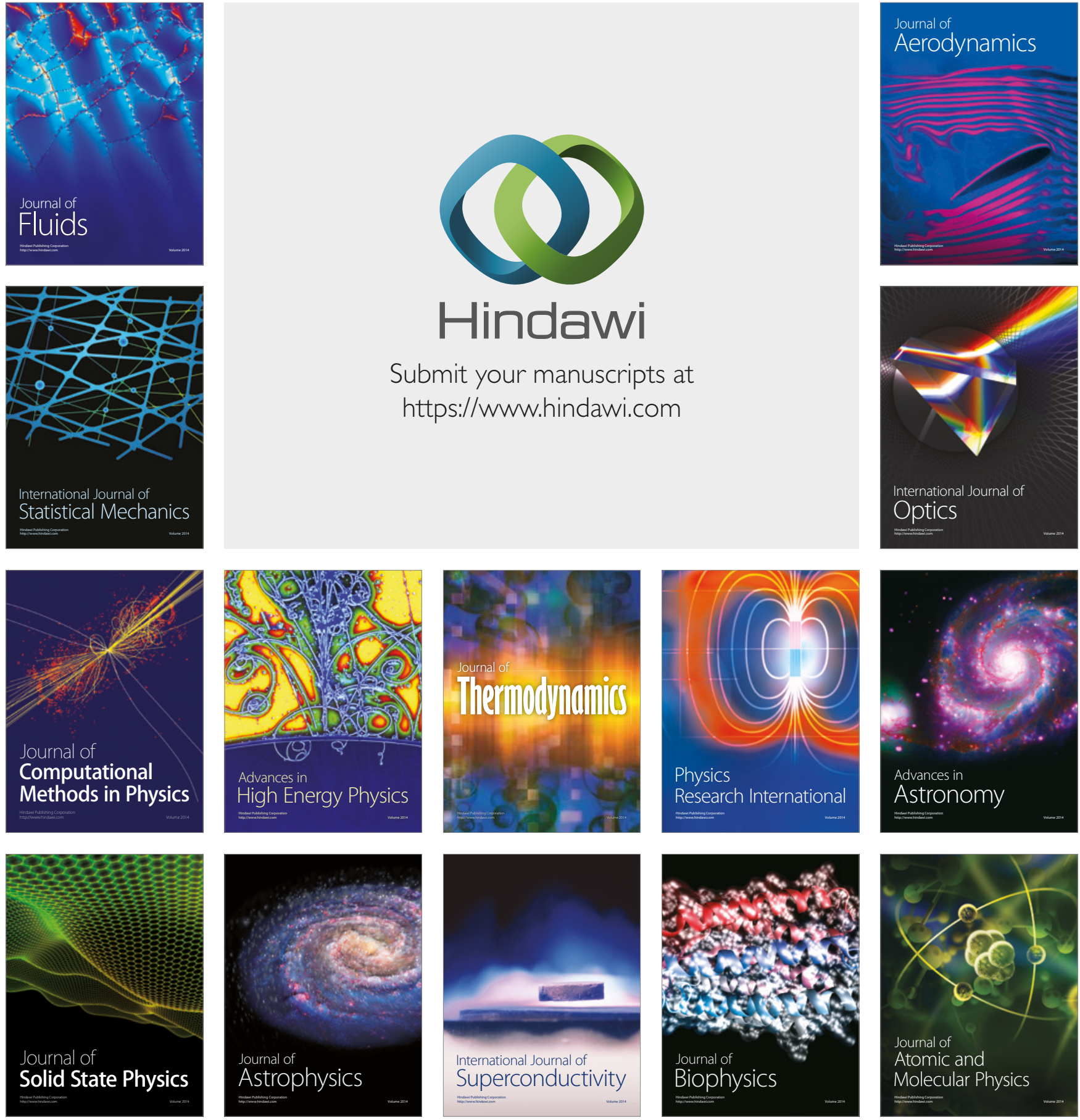